\title{
Effects of Bifidus Enhancer Yogurt on Relief from Loperamide-induced Constipation
}

\author{
Jin-Hee Choi, Seung-Hwan Jeong ${ }^{1}$, Young-Hoon $\mathrm{Cho}^{1}$, Yun-Kyung $\mathrm{Cho}^{1}$, Hae-Yeon $\mathrm{Choi}^{2}$, and Sun-Im Kim* \\ Department of Food and Nutrition, Sookmyung Women's University, Seoul 140-742, Korea \\ ${ }^{1}$ Research \& Development Center, Namyang Dairy Products Co., Ltd., Gongju 314-914, Korea \\ ${ }^{2}$ Department of Home Economics Education, Kongju National University, Gongju 314-701, Korea
}

\begin{abstract}
This study investigated the effects of bifidus enhancer yogurt (BEY) on loperamide-induced constipation in rats. The bifidus enhancer, made of rice-DDGS (Dried Distillers Grains with Solubles), improved proliferation of bifidobacteria (BB12). Male SD rats were induced with constipation using loperamide and were then used to test the effectiveness of BEY in relieving constipation. The rats were divided into four groups: normal group (NOR), loperamide-treated group (LOP), bifidus enhancer yogurt and loperamide-treated group (L-BEY), and commercial yogurt and loperamide-treated group (L-CY). Treatment of loperamide reduced the wet weight and water content of fecal pellets, but increased the number of fecal pellets in the distal colon. Meanwhile, the fecal weight of the L-BEY group showed an increase of $43 \%$ and $23 \%$ versus the LOP and L-CY group, respectively. Also, the fecal water content in the L-BEY group was $14.5 \%$ and $6.8 \%$ higher than that in the LOP and L-CY group, respectively. In addition, the L-BEY group had the fewest fecal pellets in the distal colon. In the serum lipid parameters, the LOP group had a HDL/total cholesterol ratio that was $43 \%$ lower than the NOR group, but the L-BEY group had $27 \%$ lower than NOR group. These results suggest that bifidus enhancer yogurt has superior effects when it comes to relieving loperamide-induced constipation in rats.
\end{abstract}

Key words: bifidus-enhancer yogurt, rice-DDGS, constipation, loperamide

\section{Introduction}

Constipation is prevalent in Western societies and is commonly seen as a symptom in clinical practice. In the Western world, lifestyle significantly determines health, especially later in life. A lack of physical activity, particularly if associated with over-consumption of food, can increase the risk of development of nutrition-related conditions and diseases, such as obesity, diabetes, cardiovascular disease, and constipation. Actually, constipation, which is the most frequent digestive complaint, is a symptom rather than a disease, but it is also one of the risk factors related with colorectal cancer. Around $12 \%$ of healthy people around the world have complained of constipation at some point (Wald, 2007). Its symptoms include loss of appetite, aging skin, skin ailments, headache, and abdominal inflation. Worsening symptoms can

*Corresponding author: Sun-Im Kim, Korean Food Institute, Sookmyung Women's University, Seoul 140-742, Korea. Tel: 822-710-9471, Fax: 82-2-710-9479, E-mail: sikim@sookmyung. ac.kr include hemorrhoids and terrible pain during bowel movements, and in extreme cases, it can lead to colorectal cancer (Conrazziari, 1999). Many in vitro studies and clinical trials have sought to improve the function of the gastrointestinal tract and alleviate fecal complaints with live microbial supplements (Chen et al., 2006; Shimotoyodome et al., 2001; Shin et al., 2006).

To relieve constipation and fluidize intestinal functions, it is essential to first discern the causes of the constipation and then prescribe a change of living habits and diet cure, with a particular emphasis on well-regulated meals. Foods that are effective against intestinal peristaltic motion include vegetables and marine algae, which are high in fiber; fruits containing organic acids; and dairy products like milk and yogurt, which are high in lactose (Kim et al., 2003).

Fermented milk products control intestinal functions and inhibit pathogenic bacteria because the metabolites that form during incubation of intestinal microbial metabolites impede the growth of intestinal putrefactive bacteria (Kim, 2000). Such products can also promote digestive absorption and prevent both diarrhea and constipation. In recent years, yogurt has also been reported to contribute 
to anticancer activity and a decline of serum cholesterol (Woo and Mang, 1998).

Lactobacilli and streptococci had been used to make bacillary milk. But once the beneficial effects of bifidobacteria were discovered, it became the bacteria primarily used to make fermented milk. The major metabolites of bifidobacteria are acetic acids and lactic acids. It shows greater intestinal regulation than Lactobacillus, and also decreases production of ammonia, phenol substances, and bacterial toxins in the intestine (Hughes and Hoover, 1991).

Because of bifidobacteria showing poor growth in milk and fermented milk, several substances have been studied for their potencial growth-stimulating activity. Human CMP promoted the growth of $B$. infantis, bovine casein digest and yeast extract increased the growth of various species of the genus bifidobacterium, in addition caseinomacropeptide and whey protein enhanced bifidobacteria growth in milk (Azuma et al., 1984; Janer et al., 2004; Poch and Bezkorovainy, 1988). These nutrient supplements were milk-derived components. Meanwhile, dried distillers grain is a residual product from alcohol-fermentation of cereals. Amino acids remain in dried distillers grain even after drying, so it has been used in fodder in the form of processed foods such as dried distillers grain (DDG) or dried distillers grain with soluble (DDGS) (Fontaine et al., 2007). The main ingredients of DDG are crude fat, crude protein, crude fiber, fungus bodies, and many types of minerals. Thus, DDG is a potential power and needs more research and development (Ganesan et al., 2007). DDG can be made of various ingredients, including corn, brown rice, barley, yam, and tapioca, and studies on the use of DDGS have been consistently reported (Song, 2005). However research for using DDGS in food is still currently lacking. Thus, we used rice-DDGS, an alcohol fermentation byproduct, to make yogurt with bifidus enhancer. In order to investigate how bifidus enhancer yogurt could affect the relief from constipation and intestine function, rats were induced with loperamide constipation and then given a diluted solution of yogurt.

\section{Materials and Methods}

\section{Preparation of bifidus enhancer yogurt}

The bifidus enhancer is composed of $90 \%$ rice-fermented extract and 10\% lactose. Its composition was as follows: moisture content $3.09 \%$, crude protein $9.53 \%$, crude fat $7.89 \%$, crude ash $7.99 \%$, and carbohydrate $71.5 \%$. In a preparatory experiment, bifidus enhancer made with ricefermented extract promoted growth of bifidobacteria spe- cies in yogurt. The bifidobacteria cell count of commercial yogurt (CY) and experimental yogurt 1 added bifidobacterium BB-12 were $7.5 \times 10^{5}$ and $4.0 \times 10^{6} \mathrm{CFU} / \mathrm{mL}$ respectively, but experimental yogurt 2 (BEY) added bifidus enhancer and bifidobacterium BB-12 was $5.0 \times 10^{7}$ $\mathrm{CFU} / \mathrm{mL}$ (Table 1). The yogurt used in this study was obtained by requesting products from Namyang Dairy. The commercial yogurt (CY) of the comparison group is composed of yogurt culture Yo-Mix ${ }^{\mathrm{TM}} 101$ (Danisco, Denmark), 77\% milk, 3\% skim milk, 1\% diatary fiber, and $19 \%$ any many others such as isomaltooligosaccharides, flavouring agents etc. The bifidus enhancer yogurt (BEY) of the experimental group consisted of the $\mathrm{CY}$ with the addition of $0.0075 \%$ bifidobacterium BB-12 (Chr. Hansen, Denmark), and $0.05 \%$ bifidus enhancer (S\&D, Korea).

\section{Animal care and feeding schedule}

Sprague-Dawley male rats (7-8 wk old, 220-250 g) were purchased from Central Laboratory Animal, Inc. (Seoul, Korea), and were given a 1 wk adaptation period in our lab. The rats were divided into four groups by body weight: water group (NOR), water and loperamidetreated group (LOP), bifidus enhancer yogurt and loperamide-treated group (L-BEY), and commercial yogurt and loperamide-treated group (L-CY). The rats were given two types of yogurt sample in their water for $5 \mathrm{~d}$ : $10 \%$ BEY (bifidus enhancer yogurt) and 10\% CY (Commercial Yogurt).

The other rats were given tap water. Feed (AIN-76A, Central Lab, Seoul, Korea) and water were fed ad libitum. The temperature of the lab was 20 to $22^{\circ} \mathrm{C}$, and the alternating photo cycle was $12 \mathrm{~h}$ of light and $12 \mathrm{~h}$ of darkness (Cho et al., 2008).

\section{Induction of experimental constipation}

In order to study the effect of bifidus enhancer yogurt on constipated colonic mucus, constipation was induced in the rats by subcutaneous injection of loperamide $(1.5$ $\mathrm{mg} / \mathrm{kg}$ ) twice daily (9:00 AM and 6:00 PM) for five days after the adaptation period (Jeon et al., 2007). The loper-

Table 1. Bifidobacterium species cell counts of yogurts made with and without bifidus enhancer

\begin{tabular}{cccc}
\hline \hline & $\begin{array}{c}\text { Commercial } \\
\text { Yogurt }\end{array}$ & $\begin{array}{c}\text { Experimental } \\
\text { Yogurt 1 }\end{array}$ & $\begin{array}{c}\text { Experimental } \\
\text { Yogurt 2 }\end{array}$ \\
\hline $\begin{array}{c}\text { Viable cell counts } \\
(\mathrm{CFU} / \mathrm{mL})\end{array}$ & $7.5 \times 10^{5}$ & $4.0 \times 10^{6}$ & $5.0 \times 10^{7}$ \\
\hline
\end{tabular}

Experimental Yogurt 1, commercial yogurt and $0.0075 \%$ bifidobacterium BB-12; Experimental Yogurt 2, commercial yogurt and $0.0075 \%$ bifidobacterium BB-12 and $0.05 \%$ bifidus enhancer 
amide was dissolved in $0.9 \%$ sodium chloride and was purchased from Sigma Chemical Co. (USA). Every group was treated with loperamide except the normal group (NOR group). The NOR group was given only $0.9 \%$ sodium chloride by injection.

\section{Measurement of dietary intake, water intake, body weight, feces water content and number of fecal pallets}

We measured the rats' food intake, water intake, weight of feces, and number of fecal pellets every day at 10:00 $\mathrm{AM}$, and we measured their body weight on the 1st, 7th, 12th and final day of the experiment. In order to get the dried weight of the rats' feces, the feces of the individual rats were collected and dried them in a $70^{\circ} \mathrm{C}$ oven for 24 h. We calculated the water content of the fecal pellets by the following equation: water content $=$ fecal weight dried weight/fecal weight, as used in Nakamum et al. (2001). On the final day, we sacrificed the rats to check the number of fecal pellets in the distal colon (Shimotoyodome et al., 2000).

\section{Determination of serum lipid parameters}

At the end of the experiment, blood samples were collected from all treated and untreated rats by an intracardiac puncture. Serum was separated from blood samples by centrifuging at $4000 \mathrm{rpm}$ for $10 \mathrm{~min}$ and kept at $-80^{\circ} \mathrm{C}$ for further biochemical determination. The levels of serum triglyceride (TG), total cholesterol (TC), high-density-lipoprotein cholesterol (HDL-cholesterol), and lowdensity-lipoprotein-cholesterol (LDL-cholesterol) were measured by using commercially available kits (Bio Clinical System, Korea).

\section{Statistical analysis}

All data are expressed as mean \pm SEM and were ana- lyzed using analysis of variance (ANOVA). Differences between treatments were tested using Duncan's multiple range test and are considered statistical significant at Pvalues of less than 0.05 . All statistical analyses of data were performed using an SAS statistical package (SAS Institute, 2008).

\section{Results and Discussion}

\section{Feed and water intake and body weight gain}

Table 2 shows the rats' dietary intake, water intake, and body weight gain during the study period. The dietary intake did not differ significantly among the experimental groups during the $13 \mathrm{~d}$, although the dietary intake of the loperamide-induced groups seemed to slightly decrease. The water intake differed among the groups for the $0-7 \mathrm{~d}$ period, but there was no difference in water intake during the loperamide-induced period. In addition, the rats showed similar growth in body weight before treatment with loperamide. In particular, the body weight gains of the LOP and L-CY group were significantly smaller than the NOR group during the induction of constipation. But the difference was not significant in the case of L-BEY group. Kim et al. (2008) and Shimotoyodome et al. (2000) have reported that loperamide does not significantly affect body weight gain, but the growth was still less than the control group. Also, Jeon et al. (2007) and Shin et al. (2006) have reported that the cause of the reduced body weight gain is likely the loperamide injection. The above findings agree with those previous studies. Therefore, we concluded that the decreased body weight gain was likely not due to the food intake or the yogurt, but could be attributed to the loperamide.

\section{Wet feces weight and the number of fecal pellet}

The wet feces weight of each group did not differ before

Table 2. Effects of loperamide and yogurt on food and water intake, and body weight gain

\begin{tabular}{cccccc}
\hline \hline & & \multicolumn{3}{c}{ Group $^{1)}$} \\
\cline { 3 - 6 } & & NOR & LOP & L-BEY & L-CY \\
\hline Food intake & Adaptation & $19.24 \pm 1.06^{\mathrm{a} 2)}$ & $19.07 \pm 0.98^{\mathrm{a}}$ & $18.67 \pm 1.26^{\mathrm{a}}$ & $18.64 \pm 1.33^{\mathrm{a}}$ \\
$(\mathrm{g} / \mathrm{d})$ & Constipation & $18.25 \pm 1.69^{\mathrm{a}}$ & $18.08 \pm 1.84^{\mathrm{a}}$ & $17.74 \pm 3.38^{\mathrm{a}}$ & $17.12 \pm 3.97^{\mathrm{a}}$ \\
\hline Water intake & Adaptation & $26.88 \pm 3.52^{\mathrm{ab}}$ & $28.00 \pm 3.74^{\mathrm{a}}$ & $23.13 \pm 2.75^{\mathrm{b}}$ & $24.75 \pm 3.92^{\mathrm{ab}}$ \\
$(\mathrm{g} / \mathrm{d})$ & Constipation & $31.20 \pm 4.27^{\mathrm{a}}$ & $25.40 \pm 5.03^{\mathrm{a}}$ & $28.00 \pm 4.18^{\mathrm{a}}$ & $28.20 \pm 1.92^{\mathrm{a}}$ \\
\hline Weight gain & Adaptation & $9.68 \pm 1.56$ & $9.80 \pm 0.78$ & $9.34 \pm 1.02$ & $9.76 \pm 0.49$ \\
$(\mathrm{~g})$ & Constipation & $9.21 \pm 0.85^{\mathrm{a}}$ & $6.40 \pm 1.94^{\mathrm{b}}$ & $7.76 \pm 1.20^{\mathrm{ab}}$ & $5.82 \pm 2.48^{\mathrm{b}}$ \\
\hline
\end{tabular}

${ }^{1)}$ NOR, normal group; LOP, Loperamide-treated group; L-BEY, Bifidus enhancer Yogurt+Loperamide-treated group; L-CY, Commercial

Yogurt+Loperamide-treated group

${ }^{2)}$ Values are mean \pm SD.

${ }^{\mathrm{a}, \mathrm{b}}$ Means within rows with different superscript letters are significantly different $(p<0.05)$. 


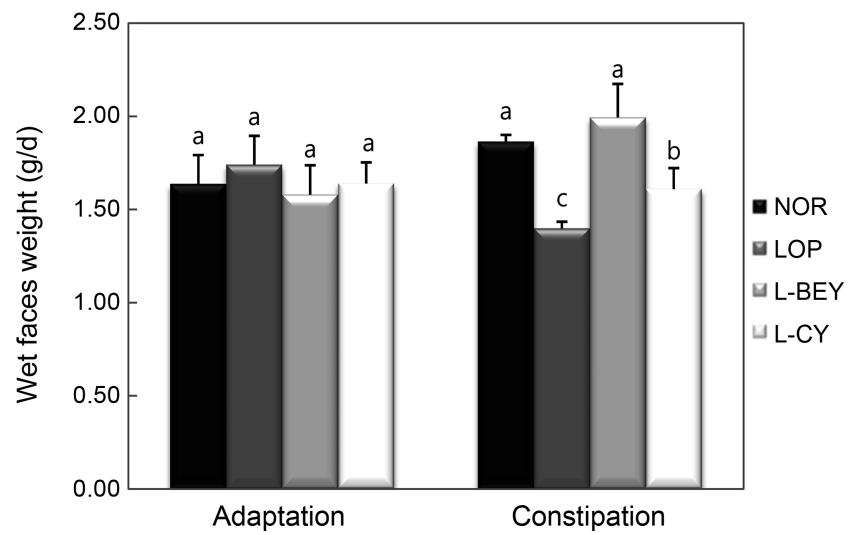

Fig. 1. Effects of Loperamide and Bifidus enhancer yogurt on wet feces weight. Adaptation, 1-wk; Constipation, loperamide-induced constipation and fed yogurt period ( 5 d); NOR, normal group; LOP, Loperamide-treated group; L-BEY, Bifidus enhancer Yogurt+Loperamide-treated group; L-CY, Commercial Yogurt+Loperamide-treated group; Bars represent mean \pm SD. ${ }^{\mathrm{a}-\mathrm{c}} \mathrm{On}$ the bars having different letters are significantly different $(p<0.05)$ by the Duncan's multiple range test.

loperamide constipation was induced, but, as shown in Fig. 1, there was a significant difference after constipation was induced and the rats were given yogurt. The feces weight of the L-BEY group who were fed bifidus enhancer yogurt increased more than the other groups, including the NOR group. The feces weight of the LOP group showed the largest drop, because of the effect of the loperamide. The fecal weight of the L-BEY group increased $43 \%$ more than the LOP group and $23 \%$ more than the LCY group. Also, the number of fecal pellets significantly changed during loperamide-induced period. The number of fecal pellets for the NOR, LOP, L-BEY, and L-CY groups were $21.1 \pm 2.3,19.0 \pm 1.1,23.3 \pm 2.7$, and $20.1 \pm 2.2$, respectively. The L-BEY group had $22 \%$ more fecal pellets than the LOP group, and approximately $9 \%$ more than the L-CY positive control group (Fig. 2). Improvement in the alleviation of constipation may be attributed to the synbiotic effect of probiotics, yam, kiwi fruit, and a soothing herb that improves the function of the gastrointestinal tract (Farombi et al., 1997; Jeon et al., 2007; Sinn et al., 2008).

\section{Feces water content}

The feces water content of all groups was quite different among each group. During the adaptation periods, the feces water content of the LOP group (which were not fed yogurt and were induced with constipation) was the highest at $27.87 \pm 3.9 \%$. The feces water percentages of the NOR, L-BEY and L-CY groups were $25.49 \pm 3.9 \%$,

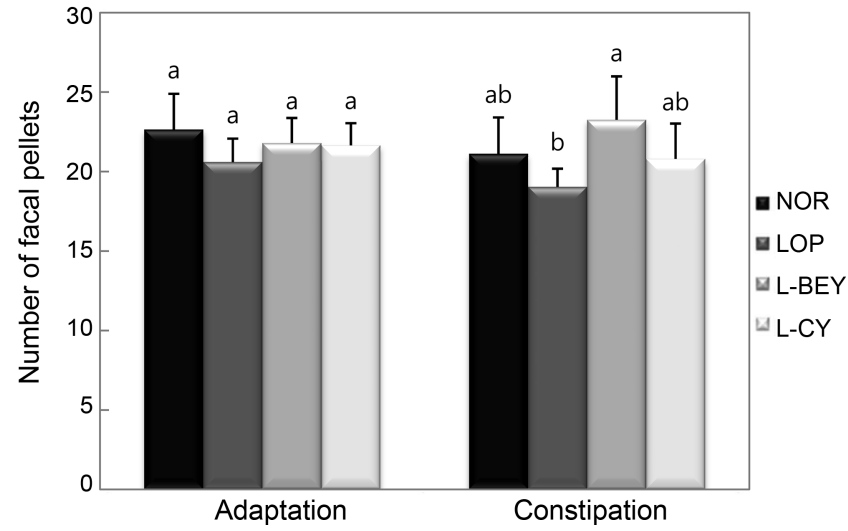

Fig. 2. Effects of Loperamide and Bifidus enhancer yogurt on the number of fecal pallets. Adaptation, 1-wk; Constipation, loperamide-induced constipation and fed yogurt period (5 d); NOR, normal group; LOP, Loperamidetreated group; L-BEY, Bifidus enhancer Yogurt+Loperamide-treated group; L-CY, Commercial Yogurt+Loperamide-treated group; Bars represent mean \pm SD. ${ }^{a, b}$ On the bars having different letters are significantly different $(p<0.05)$ by the Duncan's multiple range test.

$23.41 \pm 3.2 \%$, and $22.83 \pm 3.5 \%$, respectively (Fig. 3 ). However, those values began to change after the loperamide injection. The feces water contents of the L-BEY, L-CY, and LOP groups were $32.48 \pm 3.6 \%, 25.67 \pm 2.3 \%$, and $17.93 \pm 3.6 \%$, respectively.

The water content percentage of the L-BEY group was even higher than the NOR group $(30.23 \pm 4.4 \%)$, which were not induced with constipation, and was approximately twice as high as the LOP group. Loperamide,

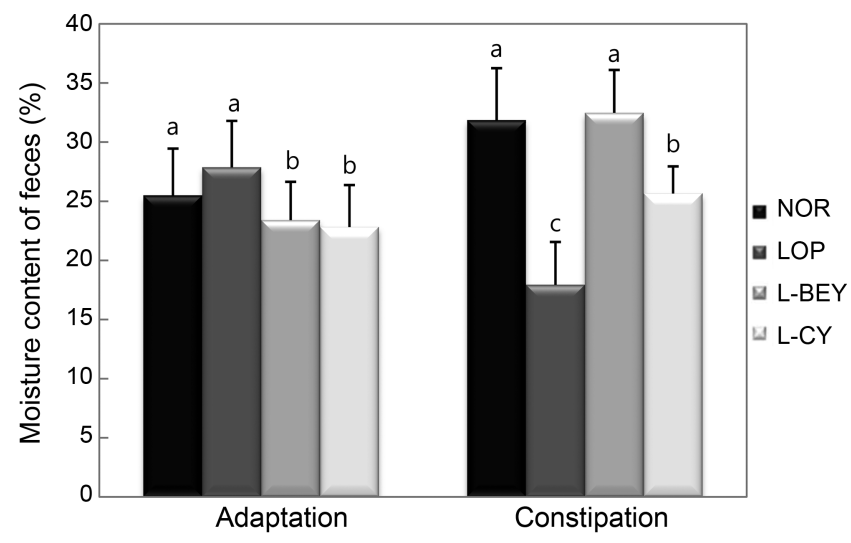

Fig. 3. Effects of Loperamide and Bifidus enhancer yogurt on moisture content of feces. Adaptation, 1-wk; Constipation, loperamide-induced constipation and fed yogurt period (5 d); NOR, normal group; LOP, Loperamide-treated group; L-BEY, Bifidus enhancer Yogurt+Loperamidetreated group; L-CY, Commercial Yogurt+Loperamidetreated group; Bars represent mean \pm SD. ${ }^{\mathrm{a}-\mathrm{c}}$ On the bars having different letters are significantly different $(p<0.05)$ by the Duncan's multiple range test. 
which was used to induce constipation on the experimental animals, is a diarrhea medicine which can repress intestinal mobility and extend the time of evacuation (Schiller et al. 1984).

In this research, the loperamide treatment reduced both the wet weight and water content of fecal pellets. The influence of loperamide was confirmed by other studies on constipation (Kim et al. 2011; Schiller et al. 1984; Shimotoyodome et al. 2000; Shin et al. 2006).

The constipation relief effects of L-BEY (bifidus enhancer yogurt) were evaluated by measuring the amount and water content of feces in rats that were fed yogurt mixed with water. Both the fecal weight and water contents of the LOP group remarkably declined in period when constipation was induced. On the other hand, the fecal weight and water contents of the L-BEY group increased significantly, more than the L-CY group.

In previous studies on how yogurt-added samples effect constipation relief (Cho et al. 2008; Jeon et al. 2007; Kim et al. 2008), the dietary fiber of the samples were a major part of the constipation relief, because the experimental samples had much more diet fiber than other samples. But the results of our study suggest that the improved relief was caused by the bifidus enhancer, which considerably increases the bifidobacteria of the yogurt, which could improve intestinal health.

\section{The number of fecal pellets in distal colon}

Loperamide increases the number of fecal pellets in the distal colon (Fig. 4). The number of fecal pellets in the distal colon of the NOR, LOP, L-BEY and L-CY groups were $3.8 \pm 1.1,5.6 \pm 1.1,3.0 \pm 1.2$ and 5.6 2.0 , respectively. In constipation, feces pellets remain in the distal colon because colon movement is reduced. However, the number of pellets in the L-BEY group was equal to the NOR group. The feces pellets of the LOP and L-CY groups were more than the others. These results suggest that bifidus enhancer yogurt has a superior effect on relieving

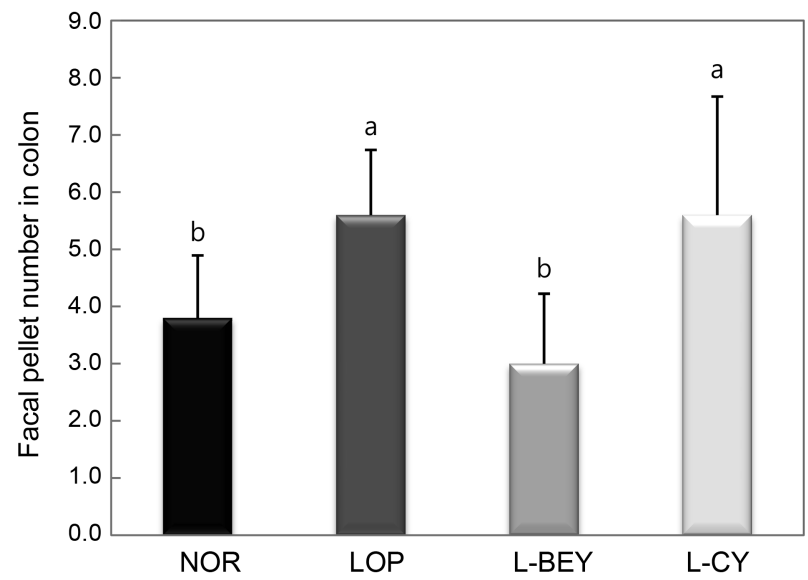

Fig. 4. Effects of Loperamide and Bifidus enhancer yogurt on the number of fecal pellets in distal colon after experiment period. NOR, normal group; LOP, Loperamide-treated group; L-BEY, Bifidus enhancer Yogurt+Loperamide-treated group; L-CY, Commercial Yogurt+Loperamide-treated group; Bars represent mean \pm SD. ${ }^{\mathrm{a}-\mathrm{c}}$ On the Bars having different letters are significantly different $(p<0.05)$ by the Duncan's multiple range test.

loperamide-induced constipation in rats. The results are similar to previous studies that examined the effects of mulberry leaves and lenitinus edodes extract on loperamide-induced constipation (Lee et al., 2008; Park et al., 2007).

\section{Serum lipids profile}

Loperamide injection induces stress and is known to alter serum lipid parameters. One mechanism by which stress may influence the lipid metabolism is by activating the sympathetic adrenal medullary axis. Epinephrine, which typically increases during periods of stress (Frankenhaeuser, 1971), may have a lipid-mobilizing effect, since infusion of epinephrine increases cholesterol levels in animals (Dimsdale et al., 1983) and enhances lipolysis in people (Freyschuss et al., 1986; Hjemdahl and Linde, 1983). Therefore, we investigated how bifidus enhancer yogurt affects the serum lipid profiles of rats with lopera-

Table 3. Effects of Bifidus enhancer yogurt on serum lipid profiles in rats

\begin{tabular}{lccccc}
\hline \hline Group $^{1)}$ & $\begin{array}{c}\text { Triglycerid } \\
\mathrm{mg} / \mathrm{dL}\end{array}$ & $\begin{array}{c}\text { Total Cholesterol } \\
\mathrm{mg} / \mathrm{dL}\end{array}$ & $\begin{array}{c}\text { HDL/Total } \\
\text { cholesterol Ratio }\end{array}$ & $\begin{array}{c}\text { LDL/Total } \\
\text { cholesterol Ratio }\end{array}$ & AI $^{2)}$ \\
\hline NOR & $146.91 \pm 12.86^{\mathrm{NS}}$ & $116.52 \pm 7.84^{\mathrm{NS}}$ & $0.48 \pm 0.12^{\mathrm{b}}$ & $0.53 \pm 0.14^{\mathrm{b}}$ & $1.15 \pm 0.08^{\mathrm{c}}$ \\
LOP & $147.40 \pm 18.02$ & $123.86 \pm 14.10$ & $0.27 \pm 0.10^{\mathrm{a}}$ & $0.75 \pm 0.08^{\mathrm{a}}$ & $2.76 \pm 0.43^{\mathrm{a}}$ \\
L-BEY & $132.62 \pm 12.83$ & $120.98 \pm 7.46$ & $0.35 \pm 0.15^{\mathrm{ab}}$ & $0.69 \pm 0.04^{\mathrm{ab}}$ & $1.83 \pm 0.67^{\mathrm{b}}$ \\
L-CY & $150.90 \pm 15.60$ & $118.64 \pm 17.21$ & $0.36 \pm 0.04^{\mathrm{ab}}$ & $0.60 \pm 0.03^{\mathrm{ab}}$ & $1.78 \pm 1.28^{\mathrm{b}}$ \\
\hline
\end{tabular}

${ }^{1)}$ NOR, normal group; LOP, Loperamide-treated group; L-BEY, Bifidus enhancer Yogurt+Loperamide-treated group; L-CY, Commercial Yogurt+Loperamide-treated group

${ }^{2)} \mathrm{AI}($ Atherogenic index): Total-HDL cholesterol/HDL

${ }^{\mathrm{a}, \mathrm{b}}$ Means within columns with different superscript letters are significantly different $(p<0.05)$. 
mide-induced constipation. Table 3 summarizes the overall data concerning triglycerides, total cholesterol (TC), HDL/TC ratio, LDL/TC ratio, and AI (atherogenic index) in the rats. In this study, the triglyceride and total cholesterol content were not significantly different among the groups, but the HDL/TC ratio was decreased in the constipation groups. The LOP group's ratio was $43 \%$ lower than the NOR group, and the L-BEY and L-CY groups were $25-27 \%$ lower than the NOR group. The LDL/TC ratio and $\mathrm{AI}$ were highest in the LOP group, but the $\mathrm{AI}$ of the L-BEY and L-CY groups (with yogurt intake) was $33-35 \%$ lower than the LOP group. Kim (2010) and Kim et al. (2005) reported that the total cholesterol content in mice increased when various types of stress were induced, but that cholesterol content was decreased by intake of pine needle yogurt and injection with Korean medicinal herbs. The results of this study indicate that the stress caused by constipation changed the cholesterol level and profiles, but the yogurt, enriched with rice-DDGS and fiber, may improve the serum lipid profile and relieve constipation.

\section{Acknowledgments}

This research was financed by the Seoul Research and Business Development program (No.10582), Republic of Korea.

\section{References}

1. Azuma, N., Yamauchi, K., and Mitsuoka, T. (1984) Bifidus growth-promoting activity of a glycomacropeptide derived from human k-casein. Agric. Biol. Chem. 48, 2159-2162.

2. Chen, H. L., Cheng, H. C., Liu, Y. J., Liu, S. Y., and Wu, W. T. (2006) Konjac acts as a natural laxative by increasing stool bulk and improving colonic ecology in healthy adults. Nutr. 22, 1112-1119.

3. Cho, Y. S., Kim, S. I., and Han, Y. S (2008) The quality characteristics of slander glasswort yogurt and its effect on the relief from constipation. Korean J. Food Cookery Sci. 24, 212-221.

4. Conrazziari, E. (1999) Need of the drug for the treatment of chronic constipation. Ital. J. Gastroenterol. Hapatol. 31, 232-233.

5. Dimsdale, J. E. and Herd, J. A (1982) Variability of plasma lipids in response to emotional arousal. Psychosomatic Med. 44, 413-430.

6. Dimsdale, J. E., Herd, J. A., and Hartley, L. H. (1983) Epinephrine mediated increases in plasma cholesterol. Psychosomatic Med. 45, 227-231.

7. Farombi, E. O., Nwankow, J. O., and Emerole, G. O. (1997) Possible modulatory effect of browned yam flour diet on chemically-induced toxicity in the rat. Food Chem. Toxicol. 35, 975-979.

8. Fontaine, J, U., Moughan, P, J., and Rutherfurd, S. M. (2007) Effect of heat damage in an autoclave on the reactive lysine contents of soy products and corn distillers dried grains with solubles. Use of the results to check on lysine damage in common qualities of these ingredients. J. Agr. Food Chem. 55, 10737-10734.

9. Frankenhaeuser, M. (1971) Behavior and circulating catecholamines. Brain Res. 31, 241-262.

10. Freyschuss, U., Hjemdahl, P., Juhlin-Dannfelt. A., and Linde, B. (1986) Cardiovascular and metabolic responses to low dose epinephrine infusion: an invasive study in humans. Clin. Sci. 70, 199-206.

11. Ganesan, V., Rosentrater, K. A., and Muthukumarappan, K. (2007) Modeling the flow properties of DDGS. Cereal Chem. 84, 556-562.

12. Hjemdahl, P. and Linde, B. (1983) Influence of circulating NE and Epi on adipose tissue, vascular resistance and lipolysis in humans. Am. J. Physiol. 245, H447-H452.

13. Hughes, D. B. and Hoover, D. (1991) Bifidobacteria; their potential for use in American dairy products. Food Technol. 54, 74-83.

14. Janer, C., Pelaez, C., and Requena, T. (2004) Caseinomacropeptide and whey protein concentrate enhance Bifidobacterium lactis growth in milk. Food Chem. 86, 263-267.

15. Jeon, J. R., Kim. J. Y., and Choi, J. H. (2007) Effect of yam yogurt on colon mucosal tissue of rats with loperamideinduced constipation. Food Sci. Biotechnol. 16, 605-609.

16. Kim, D. G., Jin, Y. G., Jin, J. Y., Kim, S. C., Kim, S. C., Han, C. H., and Lee, Y. J. (2011) Effects of the Actindia chinensis on loperamide-induced constipation in rat. Korean J. Plant Res. 24, 61-68.

17. Kim, D. H., Kim, J. H., Kim, C. H., You, J. H., Hwang, B., Lee, H. J., Kim, J. D., and Lee, H. Y. (2005) Screening of antistress activities in extracts from Korean medicinal herbs. Korean J. Med. Crop. Sci. 13, 103-109.

18. Kim, H. J., Kim. S. I., and Han, Y. S. (2008) Effects of sea tangle extract and sea tangle yogurt on constipation relief. Korean J. Food Cookery Sci. 24, 59-67.

19. Kim, H. K. (2000) Studies on the fermentation properties of yoghurt added with mulberry. MS thesis, Chungnam Nat. Univ., Daejeon, Korea.

20. Kim, I. S., Joo, E. J., Lee, K. J., and Park, E. S. (2003) Clinical nutrition and dietetic therapy. Hyoil Cultural Company, Seoul, Korea, pp. 94-107 (in Koean)

21. Kim, J. H. (2010) Immune and anti-stress activities of pine needle and the quality characteristics of pine needle yogurt. MS thesis, Sookmyung Women's Univ., Seoul, Korea.

22. Lee, J. J., Lee, Y. M., Jung, S. K., Kim, K. Y., and Lee, M. Y. (2008) Effects of dietary mulberry leaf on loperamideinduced constipation in rats. Korean J. Food Preserv. 15, 280-287.

23. Nakamum, I., Agata, G., Mizutani, M., and Lino, H. (2001) Effects of brewer's yeast cell wall on constipation and defecation in experimentally constipated rats. Biosci. Biotech. 
65, 774-780

24. Park, M. K., Jin, Y. G., Kim, D. G., Jin, J. Y., and Lee, Y. J. (2007) Effects of lenitinus edodes extract on the loperamideinduced constipation in rats. Korean J. Food Sci. Technol. 39, 88-93.

25. Poch, M. and Bezkorovainy, A. (1988) Growth-enhancing supplements for various species of the genus Bifidobacterium. J. Dairy Sci. 71, 3214-3221.

26. SAS (2008) SAS/STAT Software for PC. Release 9.2, SAS Institute Inc., Cary, NC, USA.

27. Schiller, L. R., Santa Anan, C. A., Moravski, S. C., and Fordtran, J. S. (1984) Mechanism of the antidiarrheal effect of loperamide. Gastroenterol. 86, 1475-1480.

28. Shimotoyodome, A., Meguro, S., Hase, T., Tokimitsu, I. and Sakata, T. (2000) Decreased colonic mucus in rats with loperamide-induced constipation. Comp. Biochem. Physiol. 126, 203-211.

29. Shimotoyodome, A., Meguro, S., Hase, T., Tokimitsu, I., and Sakata, T. (2001) Sulfated polysaccharides, but not cellulose, increase colonic mucus in rats with loperamide-induced constipation. Digest. Dis. Sci. 46, 1482-1489.
30. Shin, K. O., Jeon, J. R., Lee, J. S., Kim, J. Y., Lee, C. H., Kim, S. D., Yu, Y. S., and Nam, D. H. (2006) Lactic acid fermentation of Chinese yam (dioscorea batatasdecne) flour and its pharmacological effect on gastrointestinal function in rat model. Biotechnol. Bioproc. E. 11, 240-244.

31. Sinn, D. H., Song, J. H., Kim, H. J., Lee, J. H., Son, H. J., Chang, D. K., Kim, Y. H., Kim, J., J., Rhee, J. C., and Rhee, P. L. (2008) Therapeutic effect of Lactobacillus acidophilusSDC 2012, 2013 in patients with irritable bowel syndrome. Digest. Dis. Sci. 53, 2714-2718.

32. Song, M. H. (2005) Nutritional components and nutritive value of corn-DDGS about milk cow, beef cattle, pig, and fowl. Kofeed 15, 44-51.

33. Wald, A. (2007) Appropriate use of laxatives in the management of constipation. Curr. Gastroenterol. Rep. 9, 410-414.

34. Woo, S. J. and Mang, Y. S. (1998) Milk and dairy products in human nutrition. Hyoil Cultural Company, Seoul, Korea. pp. 53-65 (in Korean).

$\overline{\text { (Received 2011.9.30/Revised 2011.12.27/Accepted 2011.12.29) }}$ 